

\title{
The contribution of instructor presence to social evaluation anxiety, immersion and performance within simulation-based learning environments: a within-subject randomised cross-over trial with paramedic students
}

Brennen W Mills BHlthSc, BSc(Hons) (PubHlth) is a Research Associate ${ }^{1}$, Owen BJ Carter, BPsych, DPsych, BA, MSc (PubHlth) Associate Professor ${ }^{1}$, Nathan P Ross, BHlthSc (Paramedicine), MClinEp/MedStatc, Lecturer ${ }^{2}$, Jason K Quick, MPH, PGradDip (Intensive Care), PGradCert (Tertiary Educ), BHSc (Paramedicine), GIA (Cert), Lecturer ${ }^{3}$, Cobie J Rudd, $\mathrm{PhD}, \mathrm{MPH}, \mathrm{BH}$ thSc (Nsg), RN, GAICD, is Deputy Vice-Chancellor ${ }^{1}$, David N Reid, MHM(Hons), BSc (ParaMedSc), BAgrEc, GAICD, Senior Lecturer ${ }^{5}$.

\author{
Affiliation: \\ ${ }^{1}$ Office of the Deputy Vice-Chancellor (Strategic Partnerships), Edith Cowan University, Perth, Western Australia \\ ${ }^{2}$ School of Medicine, Deakin University, Geelong, Victoria \\ ${ }^{3}$ College of Health and Biomedicine, Victoria University, Melbourne, Victoria \\ ${ }^{4}$ School of Medical Sciences, Edith Cowan University, Perth, Western Australia \\ ${ }^{5}$ School of Medical and Health Sciences, Edith Cowan University, Perth, Western Australia
}

\section{Abstract}

\section{Background}

Previous research suggests removing instructors from simulation-based learning environments is popular with students who report greater task immersion and decreased anxiety. However, the specific impact of social evaluation anxiety on students' immersion and performance remains equivocal.

\section{Methods}

Thirty-one paramedic students completed two simulation-based clinical scenarios in random order, one in the presence of an instructor and one without. Students' distraction and time-to-completion were quantified via review of head-mounted video cameras, anxiety via continuous heart-rate (HR), and performance via two expert assessors reviewing video footage using a structured clinical assessment checklist. One-on-one, in-depth interviews followed with 12 randomly selected students.

\section{Results}

Students completed scenarios 1.8 minutes quicker when instructors were 'absent' compared to 'present' (6.6 vs. 8.4 min, p<.001), approximately half of this additional time was spent interacting with instructors $(61.4 \mathrm{sec}, \mathrm{SD}=33.4)$. Peak HR was higher in the instructor 'present' than 'absent' group (121 vs. 116 beats per minute (bpm), $p=.035$ ), but no between-group difference was found for average HR (98 vs. $100 \mathrm{bpm}, p=.407$ ). Interview data suggested students felt greater stress in the presence of instructors. No statistical difference was evident between students' performance scores in the instructor 'absent' versus 'present' conditions $(71.6 \%$ vs. $69.4 \%$ respectively, $p=.314$ ).

\section{Conclusion}

Students were more immersed, reported being less stressed and distracted, and were significantly quicker at completing clinical tasks with instructors absent, with no detriment to performance. Removing instructors during simulation-based training is likely to enhance students' immersion and potentially decrease social evaluation anxiety, but immediate performance is unlikely to improve.

\section{Keywords}

Simulation, instructor presence, paramedic education, social evaluation anxiety, immersion 


\section{Background}

Educational institutions have a responsibility to ensure health graduates entering their chosen profession are ready to practise safely (1). However, the expected level of clinical competence of newly graduated healthcare professionals often surpasses that attainable in undergraduate education alone, with previous research suggesting new graduates often lack confidence in their proficiency (2-4). Clinical training using simulationbased learning environments (SLE) are often used to help students practise translating theory into practice while removing the possibility of patient risk. However, it is noted that such experiences can be highly stressful for students and impact on their performance $(5,6)$ and therefore may not provide the optimal learning environment to develop clinical competency (7). Some suggest this is not necessarily undesirable as it emulates the pressures of true paramedic practice when patient safety is at risk (8). However, others argue that unduly stressing students during assessment is counter to the longer term educational goals of inspiring confidence, fostering learning, enhancing students' ability to self-monitor and driving self-assessment (9).

\section{Previous research suggests the human motivation to preserve} the 'social self' is similar to the desire to preserve the physical self, with individuals being prone to monitor the environment and fixate upon threats to their social wellbeing (10). The 'social evaluative stress' theory predicts that heightened evaluative stress will occur when: (\#1) a clear and important objective is sought; (\#2) the situation requires the display of valued attributes or skills; and (\#3), this attribute or skill is evaluated by others (11). Social evaluative stress has previously been confirmed in golf, public speaking and ballroom dancing, via physiological measures of salivary cortisol and heart rate, wherein its deleterious impact on performance was noted (11-13).

There is a case to be made that social evaluative stress occurs during SLE in the presence of instructors whose job is to evaluate (\#3) students skills (\#2) when attempting to achieve a clear objective (\#1). However, there are only limited published data exploring the effect of instructor presence on social evaluative stress in SLE. Quick and Ross conducted a quasi-experimental qualitative study with 30 undergraduate paramedic students and confirmed students' perceptions that the physical presence of instructors is a significant stressor (14). The authors suggested instructor presence resulted in poorer student task-specific attentiveness, consistent with other research that suggests acute stress leads to increased distractibility (15). Quick and Ross identified student-teacher discourse and a tendency for students to seek ongoing confirmation via non-verbal 'sideways glances' to the instructor as typical distractors (14). This observation is consistent with research that suggests under conditions of heightened anxiety, people's attention is biased towards the source of the threat
(16) and that if the primary stressor source is peripheral to a core task, then attention will be split between the competing tasks (17). When Quick and Ross removed instructors from the environment, students reported decreases in intimidation and self-consciousness, and a greater sense of 'ownership' of the clinical scenario. Quick and Ross concluded student performance appears to be affected by the presence of an instructor, not only due to social assessment anxiety, but also a tendency for student-teacher discourse to disrupt the continuity of and immersion within a scenario (14).

A Korean study by Bang attempted to objectively substantiate the subjective findings of Quick and Ross via an experiment separating a cohort of 83 fourth-year medical school students into instructor 'present' and 'absent' groups before undertaking a clinical task (18). Consistent with Quick and Ross, in the instructor 'absent' condition students described the scenario as being more 'realistic' and were more likely to request similar arrangements in the future. However, contradicting the predictions of Quick and Ross, no quantifiable differences in mean performance scores were detected between the two study groups, suggesting that while students may prefer instructors being removed from SLE, ultimately the presence of instructors did not impact their overall performance (18). However, the inconsistency between the two studies may be explained by the subjective, qualitative methodology used by Quick and Ross, compared to the more objective, quantitative methodology used by Bang; although, it may also be explained by the confounders in Bang's study. In the instructor 'present' condition, clinical assessments were conducted in-situ but in the instructor 'absent' condition, assessments were conducted via video-recordings of students' performance. Vivekananda-Schmidt et al. investigated the reliability of video-recorded compared to in-situ objective structured clinical examinations (OSCEs) and reported only a 'moderate' association at best ( $\mathrm{k}=.43-.51)$ (19). Consequently, Bang's study was potentially confounded by the differing methodologies used to assess students' performance in the instructor present versus absent groups.

Thus, the evidence to date consistently suggests that the presence of instructors provides distraction and increased anxiety for students when undertaking simulation-based learning. However, the extent to which this impacts on students' actual performance remains equivocal. We sought to conduct a methodologically rigorous investigation avoiding the limitations of previous studies. To do this we utilised a within-subject, randomised cross-over design to test the following hypotheses: Compared to clinical scenarios with an instructor present, assessments with an instructor 'absent' will:

H1: Decrease students' social evaluation anxiety H2: Increase students' immersion within the task H3: Facilitate better student performance. 
Mills: The contribution of instructor presence to social evaluation anxiety trial Australasian Journal of Paramedicine: 2016;13(2)

\section{Ethics}

Ethics approval was granted by Edith Cowan University Human Research Ethics Committee (\#10749).

\section{Methods}

\section{Participants}

Participants were second-year paramedicine students from Edith Cowan University (Perth, Western Australia) enrolled in a 13-week, semester-long clinical skills unit. The unit comprised weekly online, clinical theory modules, plus a 2-week intensive simulation-based workshop to provide experiential learning of advanced life support and critical care skills, reflective practice and management plans. During the 2-week workshop, students practised in small groups with two clinical instructors moving between groups to provide guidance and feedback as necessary. All students enrolled in this unit were offered the opportunity to participate in the study, occurring on the second last day of the workshop, with the incentive that they could gain additional practice before clinical examinations occurring the next day. Thirty-three students were enrolled in the unit and all agreed to participate in the scenarios. However, two preferred for their performance not to be recorded. Thus, data were collected for 31 of 33 students, representing a consent rate of $94 \%$. Participants for whom data were collected had an average age of 27.6 years $(S D=8.4)$ with $71 \%$ being female.

\section{Materials \\ Clinical Scenarios}

By consensus, paramedic teaching staff selected six scenarios deemed to be within the clinical capacity of secondyear paramedicine students but sufficiently challenging to discriminate between low and high levels of competency. These included patient management of burns, diabetes, life-threatening haemorrhaging, orthopaedic trauma, severe asthma and cerebrovascular disease. Each scenario was designed to involve a live patient-actor able to provide all clinically relevant information necessitating no need for the participant to communicate with the instructor.

\section{Measures}

\section{Cardiovascular reactivity}

Based on the method used by Hardy et al. (12), students wore Polar s610i watches and chest straps (Polar, Kempele, Finland) to record continuous heart-rate data at 5 second intervals in beats per minute (bpm) for the duration of each scenario. Cardiovascular reactivity has previously been demonstrated as a robust measure of arousal in sedentary simulated environments (eg. driving- and flight-simulators) (20). However, our students were physically engaged within their simulated environment, potentially introducing confounders to the HR data. As such, we deliberately kept the requirement for physical engagement equal between comparison groups, with instructor presence or absence being the only manipulation between experimental conditions. Hence, we reasoned that any differences in HR reactivity between instructor 'present' and 'absent' conditions could be reasonably attributed to differences in psychological state reflecting arousal due to the participants' mental and/or emotional reactions.

\section{Student-instructor interactions}

Sony Action Cams (Sony, Tokyo, Japan) were strapped to the heads of students to allow video-capture from a firstperson point-of-view for each scenario. Time students spent conversing with instructors was coded in seconds. Also coded was the number and duration of 'sideways' glances directed by students towards instructors for non-verbal confirmation, as described by Quick and Ross (14).

\section{Time-to-completion}

Action Cam video-recordings were used to quantify timeto-completion in seconds, operationalised as the time when students first entered the scenario to when instructors terminated scenarios, typically the point where students had completed patient treatment and had called for transport.

\section{Structured clinical assessment checklist}

A draft structured clinical assessment checklist was devised, based on different aspects of the 11 capacity development areas outlined by the Australian Council of Ambulance Authorities Paramedic Professional Competency Standards (v.2) (21). A panel of senior paramedicine clinical staff at Edith Cowan University checked these items to confirm their content validity. This resulted in a final check list of 15 items, with students scored as either 2 (competent), 1 (developing) or 0 (supervision required) for each item, resulting in scores from $0-30$, which was then converted to a percentage.

\section{Procedure}

From the Edith Cowan University Health Simulation Centre we commandeered two simulation suites equipped with twoway mirrors to adjoining control booths and two non-mirrored debriefing rooms. Within each room scenarios were enacted by live-actor-patients working from standardised scripts. Actor-patients received one day's prior training from paramedic teaching staff. Students were randomly assigned into groups of three following the Physician Model of Assessment that separates students into 'delegator' and 'respondents' (22). At the commencement of scenarios, students were provided 'call-out' information before entering the room with the actorpatient. The delegator wore the headcam and HR monitor in each scenario and was responsible for all clinical decisionmaking and delegation of tasks to the two respondents (eg. 'take blood pressure', 'apply splint', 'administer salbutamol'). The assessment works on the assumption that this frees delegators to focus on higher-order tasks such as overall patient management, communication, critical thinking and clinical reasoning, and is therefore a better reflection of overall clinical competency (22). 
Students remained in the same group for all six randomly ordered scenarios, with each student rotating between delegator (twice) and respondents (four times). As delegator, each student undertook one scenario with an instructor 'present' and one with instructor 'absent', in a random order. In the instructor 'present' condition, scenarios were undertaken in the debriefing rooms with the same clinical supervisors who had been teaching the students for the past fortnight, positioning themselves behind the student to ostensibly assess performance on a clipboard. In the instructor 'absent' condition, scenarios occurred in the simulation suites and students were informed by instructors prior to commencement that their performance would be viewed from the control booth behind the two-way mirror. In this fashion, the knowledge that participants were being observed and assessed by an instructor was kept constant between study conditions, thereby isolating the physical presence or absence of the instructor in the room as the sole independent variable.

As stated, all necessary information to complete each clinical assessment was available to students without the need to ask the instructor. However, if students sought clarification about the scenario from instructors in the 'present' condition, instructors were permitted to respond but were instructed to keep responses brief and to avoid providing feedback on performance.

In order to avoid the potential confounding of different assessment methods suffered by Bang's study, students' performances in both 'present' and 'absent' conditions were assessed in a consistent manner by review of their headcam footage. Two expert instructors from interstate, each with a minimum of 15 years paramedic experience, met to agree on a consistent marking strategy for each item of the structured clinical assessment checklist. They then independently viewed randomly ordered headcam footage and assessed students' performance based on the checklist. The two experts then met again to compare scores and conferred to arrive at a consensus for each student's performance.

\section{Statistical analysis}

Paired samples t-tests compared each student's average and peak HR, time-to-completion and performance measures in the instructor 'present' versus 'absent' conditions. We used $G^{*}$ Power (v.3.1) to estimate that our sample size of $n=31$ would be sufficient to detect an effect size of Cohen's $d=0.8$ ( $\alpha=.05$, $\beta=.20$ ); being the equivalent to a difference between group means of $7 \mathrm{bpm}(\mathrm{SD}=10)$ for our HR measure and 1 minute $(S D=1.5)$ for our time-to-termination measure.

\section{Interviews}

A random selection of students $(n=12)$ were individually interviewed the next day, equally stratified by their order of 'absent' versus 'present' conditions. A pragmatic, actionresearch oriented, interpretive inquiry approach (15) was combined with the queued retrospective verbalisation method $(23,24)$, where students reviewed their own video footage and were asked to recall their immediate experiences, feelings, beliefs and perceptions at each stage of the scenario. Student interviews were video-recorded and transcribed verbatim. QST NVivo software was used to organise text, coding and theme identification. Bracketing techniques were used during coding to suspend researcher biases and presuppositions (25). To ensure rigour, two researchers independently reviewed and coded all 12 interviews before meeting together to compare notes and arrive at a final consensus on coding and theme identification.

\section{Results}

\section{Cardiovascular reactivity}

No significant within-subject differences were found between instructor 'present' and 'absent' conditions for average HR throughout clinical scenarios (98 vs. 100 bpm respectively, $\mathrm{t}(26)=-.843, \mathrm{p}=.407)$. However, a statistically significant difference was noted in the within-subject peak HR between 'present' and 'absent' conditions (121 vs. 116 bpm respectively, $t(26)=2.226, p=.035)$. In both conditions, peak HR occurred within the first minute of the scenario (see Figure 1).

\section{Time-to-completion and student-instructor interactions}

Students average time-to-completion was 1.8 minutes quicker in their instructor 'absent' compared to their 'present' conditions ( 6.6 vs. 8.4 minutes respectively, $t(29)=4.092, p<.001$ ). Just over half $(57 \%)$ of this additional time was spent interacting with the instructor (61.4 seconds, SD=33.4), of which approximately equal time was spent providing an ongoing narration to the instructor of the students' clinical reasoning (27.9 seconds, $\mathrm{SD}=27.4$ ) and the students seeking clarification about the scenario from the instructor (28.2 seconds, SD=16.7). The remaining 5.3 seconds ( $S D=4.3$ ) was accounted for by students making an average of three non-verbal 'sideways' glances toward instructors.

\section{Performance}

An examination of pooled clinical assessment scores awarded by the independent assessors suggested the distribution of scores met assumptions of normality (skewness $=-.041$, kurtosis=-.384). No statistical difference was detected between students mean scores for their instructor 'absent' versus 'present' conditions $(71.6 \%$ vs. $69.4 \%$ respectively, $t(30)=1.025$, $p=.314)$.

\section{Interviews}

Four consistent themes arose during analysis of the interviews, namely 'social anxiety', 'inhibited performance', 'distraction' and 'scenario ownership'. 


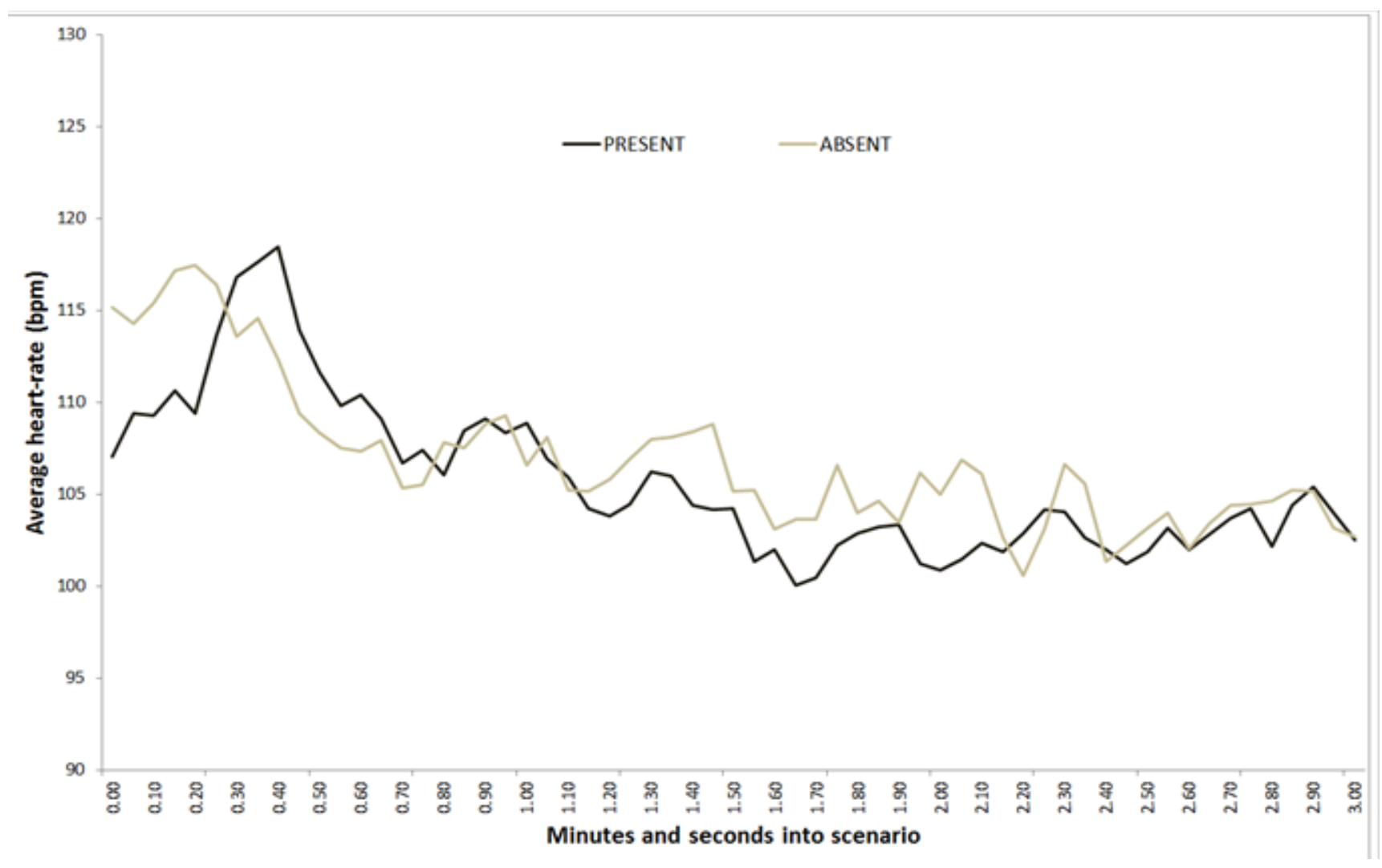

Figure 1. Within-subject differences in average heart rate in 5 -second epochs by experimental condition

\section{Social anxiety}

Students frequently suggested the presence of an instructor made them more anxious, eg. 'The first scenario without the instructor being there was less stressful' and 'I was a bit nervous because he [the instructor] was watching - they expect a lot from us I think so it can be a bit scary doing it in front of them'. This encompassed a preoccupation with being judged, eg. 'They're professionals and they tell me off if I do things wrong, or if I don't listen, or don't do what I need to do' and 'Because the teacher was there I was like, 'oh my God, don't screw up, don't screw up!" This anxiety often stemmed from a desire to not disappoint their instructor, eg. 'You want to do the best you can and want them to respect you. Even though she's [instructor] really nice, she's really scary' and 'I thought it was a stroke at this point so I was going down that path, but never verbalised that to him [instructor] so was shit-scared I was going to get into trouble'.

\section{Inhibited performance}

Some commented on how this anxiety adversely affected their ability to think, in turn affecting their performance in the scenario, eg. 'When you know you're being judged and assessed on it I get stunned like a deer in the headlights and my heart-rate spikes and I think 'oh God!" and 'Because she [instructor] was there I needed to stop a couple of times and think 'OK, what am I doing? What do I need to think about? What've I done already?' However, some participants (typically older) welcomed instructor scrutiny as motivation to perform, eg. 'It's good having someone watching because they'll tell you what you did wrong and stuff like that' and 'For me the instructor is just like a machine giving me information - I don't care what they think -1 just need that information to get better.' Notwithstanding, the majority of participants preferred no instructor being present in the room, eg. 'I suppose I was glad that I didn't have an instructor with me'. Reasons for this included students feeling more confident in their abilities, eg. 'I think I'm more confident within myself when I don't have someone watching'.

\section{Distraction}

Students also suggested it was less distracting in the instructor absent condition, eg. 'Him [instructor] not being there made it easier because I could think about what I wanted to do without being distracted' and 'I didn't have that feeling in the back of my brain - you know - knowing that someone's watching my performance - I sort of forgot about everything else and just focussed'.

\section{Scenario ownership}

Furthermore, students expressed satisfaction when completing a scenario on their own, eg. 'Without having the instructor there I thought 'OK, there were probably things I missed because I'm still learning, but hey, I did it all myself without anyone looking over my shoulder'. It allowed me to process what happened instead of stew over what he [instructor] was going to say' and 'Without having the instructors there, it allowed me to process what they'd taught us and then consolidate, whereas if I had an instructor there I would've felt like I was being taught more things and I would have got flustered.' 


\section{Discussion}

The present study contributes to two previous investigations of the effect of instructor presence versus absence on the social evaluative anxiety, immersion and performance of students during simulation-based training in health $(14,18)$. Our mixed-methods approach replicated both the qualitative and quantitative methods of the previous studies but to this we added the new measure of HR to provide an objective measure associated with social evaluation stress - subjectively reported in both previous studies - and we also quantified student interaction with instructors; not attempted by either previous study. We also improved on Bang's quantitative methodology by ensuring students' performances were judged by the same instructors in both 'present' and 'absent' conditions using a consistent, video-capture and assessment method; thereby removing the potential confounder of differing assessment methods. Our study was further strengthened by the use of a within-subject, randomised cross-over design such that our comparisons were not confounded by between-subject random effects associated with students differing cardiovascular health or clinical abilities, such as was the case with Bang (18).

Our first hypothesis predicted less stress among students when instructors were absent compared to present, based on the assumption that assessor presence is associated with social evaluation anxiety. The qualitative findings confirmed that many students felt heightened anxiety in the presence - compared to absence - of instructors and a preference for the instructor absent condition, consistent with previous research $(14,18)$. Although a few students described heightened motivation in the presence of instructors - a good thing that might also be associated with arousal - such students were in the minority with most clearly preferring the instructor 'absent' condition. Qualitative findings were corroborated by students' peak HR data, which was higher when instructors were present. However, students' HR averages - which for the present study we ultimately view as a more holistic demonstration of arousal - were no different between conditions. Taken together our first hypothesis was supported by subjective data but only partial support was provided by objective measures. Thus, we conclude only partial support was found for our first hypothesis through our lesser objective measure (peak HR) and subjective report via qualitative interviews.

Our second hypothesis predicted students would be distracted by the presence of the instructor, and that removal of the instructor would heighten students immersion in scenarios and allow them to 'narrow' their focus toward the core task (ie. treatment of the patient) during training. This was based upon previous literature regarding the effects of anxiety on distractibility and attention (15) as well as the observations of Quick and Ross that instructor presence leads to studentteacher discourse that disrupts the continuity of a clinical scenario (14) and the similar claim by Bang's students that instructor presence decreases the 'realism', or ability of students to 'immerse' themselves (ie. suspension of disbelief) in clinical scenarios (18). Our data clearly supported this hypothesis by demonstrating students spent an average of just over one minute conversing with the instructor when present, accounting for just over half the amount of time longer it took students to complete their scenario in comparison to their 'absent' condition. Around half this time was spent seeking clarification from the instructor but it is interesting to note that students could have just as easily asked the actor-patient for the same information; as they were forced to do in the instructor 'absent' condition. Having the option of seeking clarification from an instructor is inherently unrealistic, further reflecting students lowered immersion in the 'present' condition. Time-to-completion data also suggests lowered immersion with instructors present, with students performing clinical tasks significantly quicker when instructors were removed. Many students confirmed the removal of the instructor allowed them to narrow their focus toward treatment of the patient, even though they were still aware of being viewed by instructors through the two-way mirror. Our objective 'sideways glance' data also confirmed Quick and Ross' suggestion that students seek ongoing, non-verbal feedback from instructors (14). It appears that the visible presence of the instructor served as a constant reminder and distraction to students of being evaluated to the point they felt as though they were being 'assessed', and therefore ensured stringent application of systematic processes at the expense of swift treatment. While this is not necessarily an indication of diminished learning, it is likely an indicator of decreased immersion. The students descriptions of becoming anxious and 'flustered' in the presence of instructors, thereby adversely affecting their ability to think, is also consistent with previous literature suggesting selective attention (ie. one's ability to focus on the core task and filter out extraneous information) is diminished under conditions of heightened anxiety (17).

Finally, we predicted the removal of instructors would facilitate heightened performance. This hypothesis was not supported by our assessment data - no statistically significant differences were noted between students' scores in the 'present' versus 'absent' conditions. However, in the face of equivalent performances in both conditions, we are compelled to consider the time-tocompletion measure, which clearly was quicker for students in the instructor 'absent' compared to 'present' conditions - but with no apparent degradation to clinical decision-making. Around half of the extra time taken in the instructor 'present' condition was attributable to student-teacher discourse. Qualitative data suggested the other half was likely accounted for by students second-guessing themselves when instructors were present. Thus, it appears our students were contending with dual aspects of the scenario when an instructor was present - clinical-decision making and the ongoing reminder that their performance was being evaluated. These conclusions certainly align with previous research that suggests attention is biased toward threatening cues (in this case the clinical instructor making judgements on clinical performance) (16) and that under high anxiety conditions it becomes more difficult to filter out extraneous information affecting our ability to focus on the central/core task $(17,26)$. 
To summarise, hypothesis one (social evaluation anxiety) was partially supported, hypothesis two (immersion) was clearly supported and hypothesis three (performance) was not supported. When considering these results it is important to consider the study's limitations. For example, we used a convenience sample of paramedicine students meaning the generalisability of our research is limited. Future research should attempt to replicate our study findings with other samples across multiple disciplines at different stages of learning. Also, our assessments were conducted under relatively 'low stakes' conditions. It would be of interest to repeat this study during a 'high stakes' assessment to investigate the effect of assessor presence on anxiety, distraction and performance. Our data would predict no significant difference in performance if the assessor were removed. However, the heightened anxiety associated with fear-of-failure (27) may be more sensitive to assessor absence versus presence than was the case in our study. This is an avenue for future research. Also, it is possible $\mathrm{HR}$ data was misinterpreted as anxiety as $\mathrm{HR}$ also increases due to excitement and physical exertion. However, we are confident in our decision to attribute any between-group differences found in HR to increased anxiety as scenario physical requirements remained constant across our two study conditions, thus leaving changes in HR likely attributable to social processes alone. As mentioned, we only observed differences in peak HR between study conditions, but not for average HR - the measure we place more credence to as a true reflection of increased arousal as it takes into account the scenario in its entirety. While peak HR is often used to infer level of anxiety in the psychophysiology literature (eg. 28,29), we maintain only partial support for our first study hypothesis when also considering the qualitative data that certainly suggests students associated the instructor 'present' condition with heightened anxiety.

These limitations notwithstanding, our results lead us to conclude that the presence of an instructor during simulation-based training is clearly distracting to students, leads to heightened subjective anxiety for many, decreases students' 'immersion' in the scenario and impacts on the timeliness of their performance, but does not increase performance errors per se. Our results suggest removing instructors from view during simulation-based training would be popular with students, possibly decrease their anxiety, increase buy-in, and facilitate a more timely performance of clinical skills without adversely affecting overall performance by allowing students to focus on the treatment of the patient, rather than be preoccupied by their personal performance as perceptually judged by their instructor. As others have pointed out, training in clinical education should foster learning, inspire confidence, enhance the learner's ability to self-monitor and drive self-assessment (9). Removing instructors from sight during clinical training in simulation is entirely consistent with these noble goals.

Future replications of this study might consider other, more sensitive, objective measures of anxiety, perhaps via cortisol analyses. Cortisol from saliva or blood samples is a well- established measure of anxiety (30) and has been used as an objective measure of stress in a number of health simulationbased studies (31-33) with one suggesting cortisol levels measured during high fidelity SLE were no different to those measured in a real-life operating room (34). Also, in the present study we found increased immersion did not affect immediate performance, but previous research suggests greater immersion is espoused as increasing transfer of knowledge to real world environments $(35,36)$. As this was beyond the scope of the present study, future research could investigate how instructor presence in SLE impacts on learning outcomes retention and transfer.

\section{Conflict of interest}

The authors declare they have no competing interests. Each author of this paper has completed the ICMJE conflict of interest statement.

\section{Funding}

This project was funded by the Australian Government Department of Health.

\section{References}

1. Tavares W, Boet S, Theriault R, Mallette T, Eva K. Global rating scale for the assessment of paramedic clinical competence. Prehosp Emerg Care 2012;17:57-67.

2. Han W, Maxwell S. Are medical students adequately trained to prescribe at the point of graduation? Views of first year foundation doctors. Scott Med J 2006;51(4):27-32.

3. Hodgetts S, Hollis V, Triska O, Dennis S, Madill H, Taylor E. Occupational therapy students' and graduates' satisfaction with professional education and preparedness for practice. Can J Occup Ther 2007;74(3):148-60.

4. Romyn D, Linton N, Giblin C, et al. Successful transition of the new graduate nurse. Int J Nurs Educ Scholarsh 2009;6(1):34.

5. Alinier G, Hunt B, Gordon R, Harwood C. Effectiveness of intermediate-fidelity simulation training technology in undergraduate nursing education. Journal of Advanced Nursing 2006;54(3):359-69.

6. Levett-Jones T, Gersbach J, Arthur C, Roche J. Implementing a clinical competency assessment model that promotes clinical reflection and ensures nursing graduates' readiness for professional practice. Nurse Educ Pract 2011;11:64-9.

7. Rushforth $\mathrm{H}$. Objective structured clinical examination (OSCE): Review of literature and implications for nursing education. Nurse Educ Today 2007;27:481-90.

8. Ruesseler M, Weinlich M, Byhahn C, et al. Increased authenticity in practical assessmetn using emergency case OSCE stations. Adv Health Sci Educ Theory Pract 2010;15:81-95. 


\section{References (cont.)}

9. Epstein R, Hundert E. Defining and assessing professional competence. JAMA 2002;287:226-35.

10. Dickerson S, Kemeny M. Acute stressors and cortisol responses: a theoretical integration and synthesis of laboratory research. Psychol Bull 2004;130:355-91.

11. Rohleder N, Beulen S, Chen E, Wolf J, Kirschbaum C. Stress on the dance floor: the cortisol stress response to social-evaluative threat in competitive ballroom dancers. Pers Soc Psychol Bull 2007;33(1):69-84.

12. Hardy L, Mullen R, Jones G. Knowledge and conscious control of motor actions under stress. Br J Psychol 1996;87:621.

13.Dickerson S, Myceck P, Zaldivar F. Negative social evaluation, but not mere social presence, elicits cortisol responses to a laboratory stressor task. Health Psychol 2008;27(1):116-21.

14. Quick J, Ross N. Does removing the clinician from the immediate vicinity of high-fidelity simulation impact on student outcomes and learning experiences? Poster presentation: Asia Pacific Meeting on Simulation in Health, Hong Kong. September, 2011.

15. Skosnik P, Chatterton R, Swisher T, Park S. Modulation of attentional inhibition by norepinephrine and cortisol after psychological stress. Int J Psychophysiol 2000;36:59-68.

16. MacLeod C, Rutherford E. Anxiety and the selective processing of emtional information: mediating roles of awareness, trait and state variables, and personal relevance of stimulus materials. Behav Res Ther 1992;30:479-91.

17. LeBlanc $V$. the effects of acute stress on performance: implications for health professions education. Acad Med 2009;84(10):S25-33.

18. Bang J. Effects of rater's presence in OSCE station on examinee's scores and performance. Korean J Med Educ 2008;20(3):241-7.

19. Vivekananda-Schmidt P, Lewis M, Coady D, et al. Exploring the use of videotaped objective structured clinical examination in the assessment of joint examination skills of medical students. Arthritis Care Res 2007;57(5):869-76.

20.Jang D, Kim I, Nam S, Wiederhold B, Wiederhold M, Kim S. Analysis of physiological response to two virtual environments: driving and flying simulation. Cyberpsychol Behav 2002;5(1):11-8.

21.Papp I, Markkanen $M$, von Bonsdorff M. Clinical environment as a learning environment: student nurses' perceptions concerning clinical learning experiences. Nurse Educ Today 2003;23(4):262-8.

22. Quick J. Physician's model for clinical scenarios - an innovative approach to paramedic education and assessment. Response, Spring Edition, Melbourne, Australia. 2012.
23. Holmqvist K, Nyström M, Andersson R, Dewhurst R, Jarodzka I, can de Wejjer J. Eye tracking: a comprehensive guide to methods and measures. New York: Oxford University Press; 2011.

24. van den Haak M, De Jong M, Schellens P. Retrospective vs. concurrent think-aloud protocols: testing the useability of an online library catalogue. Behav Inf Technol 2003;22(5):33951.

25. Creswell J. Research design: qualitative, quantitative, and mixed methods approaches (third edition). Thousand Oaks, Calif: Sage Publications. 2009.

26. Braunstein-Bercovitz H. Does stress enhance or impair selective attention? The effects of stress and perceptual load on negative priming? Anxiety Stress Coping 2003;16:345-57.

27. Conroy D, Willow J, Metzler J. Multidimensional fear of failure measurement: The Performance Failure Appraisal Inventory. J Appl Sport Psychol 2002;14:76-90.

28. Childs E, Vicini L, De Wit H. Responses to the Trier Social Stress Test (TSST) in single versus grouped participants. Psychophysiol 2006;43(4):366-71.

29. Kirschbaum C, Schommer N, Federenko I, et al. Short-term estradiol treatment enhances the pituitary-adrenal axis and sympathetic responses to psychosocial stress in healthy young men. J Clin Endocrinol Metab 1996;81:10.

30. Hellhammer D, Wust S, Kudielka B. Salivary cortisol as a biomarker for stress research. Psychoeuroendocrinology 2009;34:163-71.

31.Bong C, Lightdale J, Fredette M, Weinstock P. Effects of simulation versus traditional tutorial-based training on physiologic stress among clinicians: a pilot study. Simul Healthc 2010;5(5):272-78.

32. LeBlanc V, Regehr C, Tavares W, Scott A, MacDonald R, King K. The impact of stress on paramedic performance during simulated critical events. Prehosp Disaster Med 2012;27(4):369-74.

33. Wetzel C, Black S, Hanna G, et al. The effects of stress and coping on surgical performance during simulations. Ann Surg 2010;251(1):171-76.

34. Jones T, Goss S, Weeks B, Miura H, Bassandeh D, Cheek $D$. The effects of high-fidelity simulation on salivary cortisol levels in SRNA students: a pilot study. The Scientfic World Journal 2011;11:86-92.

35. Beaubien J, Baker $\mathrm{D}$. The use of simulaton for training teamwork skills in health care: how low can you go? Qual Saf Health Care 2004;13(Suppl 1):i51-6.

36. Maran N, Glavin R. Low- to high-fidelity simulation - a continuum of medical education? Med Educ 2003;37(S1):22-8. 